\title{
Unethical informed consent caused by overlooking poorly measured nocebo effects
}

\author{
Word Count
}

3872 (excluding references)

\section{Key Words}

Nocebo, placebo, informed consent, ethics, non-maleficence, autonomy, justice, evidence-based medicine

\section{Please cite this article as}

Howick J. Unethical informed consent caused by overlooking poorly measured nocebo effects Journal of Medical Ethics Published Online First: 16 February 2020. doi: 10.1136/medethics-2019105903 
Howick J. Unethical informed consent caused by overlooking poorly measured nocebo effects Journal of Medical Ethics Published Online First: 16 February 2020. doi: 10.1136/medethics-2019-105903

\section{ABSTRACT}

Unlike its friendly cousin the placebo effect, the nocebo effect (the effect of expecting a negative outcome) has been almost ignored. Epistemic and ethical confusions related to its existence have gone all but unnoticed. Contrary to what is often asserted, adverse events following from taking placebo interventions are not necessarily nocebo effects; they could have arisen due to natural history. Meanwhile, ethical informed consent (in clinical trials and clinical practice) has centred almost exclusively on the need to share intervention risks with patients to preserve their autonomy. Researchers have failed to consider the harm caused by the way in which such risk information is shared. In this paper, I argue that the magnitude of nocebo effects must be measured using control groups consisting of untreated patients. And, because the nocebo effect can produce harm, the principle of non-maleficence must be taken into account alongside the principle of autonomy when obtaining (ethical) informed consent. 


\section{BACKGROUND}

Reeves, Ladner, and Hart report the case of Mr. A, who presented to the emergency room of a hospital with blood pressure of $80 / 40$ and a heartrate of 110 . Before collapsing to the floor, he reported having taken all 29 pills from a bottle that contained capsules to be taken as part of a clinical trial of a new antidepressant medication. ${ }^{1}$ Doctors were able to raise his blood pressure with intravenous saline solution (over 6 hours, he was given 6 litres of fluid). Eventually, a physician from the clinical trial arrived and ascertained that Mr. A had taken placebos rather than 'real' antidepressant drugs. Upon being informed of this, Mr. A expressed tearful relief, and within 15 minutes his blood pressure and heartrate returned to normal. Reeves et al. claim this is an example of the nocebo effect, ${ }^{1}$ which I will provisionally define as follows:

Nocebo effect: adverse event arising due to beliefs or expectations of a patient

Unlike the placebo effect, the nocebo effect has received relatively little attention (see Figure 1). Meanwhile, as I will show, intertwined epistemological and ethical confusions surround the nocebo effect. Nocebo effect estimates have failed to control for natural history, and evaluations of ethical informed consent have very rarely taken nocebo effects into account. ${ }^{2}$ Whereas the ethics of placebo effects is controversial, ${ }^{34}$ unnecessary nocebo effects, if they can be avoided, seem harms that should be avoided.

Before proceeding, I will make a remark about the scope of this paper. While I focus on nocebo effects (and methods to mitigate them) in clinical trials, I will also use some examples from clinical practice. My working assumption will be that epistemological and ethical issues surrounding nocebo effects are sufficiently similar in trials and practice to discuss them together. Given the lack of attention nocebo effects have received, it is useful to clarify the important philosophical issues in a general way, leaving a more detailed discussion of what I suspect are subtle (yet important) differences between nocebo effects in trials and practice to future work.

\section{[Figure 1 about here]}

\section{DISTINGUISHING MISATTRIBUTED ADVERSE EVENTS FROM NOCEBO EFFECTS WITHIN CLINICAL TRIALS}

\footnotetext{
${ }^{1}$ Mr. A's state of mind leading up to his decision to 'overdose' (resulting in the release of catecholamines) likely exacerbated the situation.
} 
Howick J. Unethical informed consent caused by overlooking poorly measured nocebo effects Journal of Medical Ethics Published Online First: 16 February 2020. doi: 10.1136/medethics-2019-105903

Beecher's classic 1955 paper The Powerful Placebo (one of the earliest examples of a systematic review) found that a third of people who took placebos recovered, and concluded that placebos were powerful. ${ }^{5}$ However, patients may have recovered even had they not taken the placebo. Hence, Beecher committed the post hoc ergo proctor hoc fallacy. To be fair to Beecher, he discusses this possibility, and dismisses it as an unlikely complete explanation for the effect magnitude he found.

In 1998, Kirsch and Sapirstein distinguished between placebo response (change after taking a placebo) and placebo effect (component of the change caused by the placebo). To measure placebo effects, Hróbjartsson and Gøtzsche compared outcomes in placebo groups with outcomes in 'untreated' groups. (Aside: I will leave the evidence that so-called 'untreated groups' are not untreated. So-called untreated groups usually have diagnostic tests, have routine contact with healthcare providers, and hear about how wonderful the treatment is from the staff. Given all the intervention that the waiting list group receives, it is unsurprising that Krogsboll and colleagues found that patients in 'untreated' groups experienced between $20 \%$ and $24 \%$ improvement while they were being untreated. ${ }^{6}$ The treatment of so-called untreated groups introduces bias to Hróbjartsson and Gøtzsche's study that is beyond the scope of this paper to discuss in detail. Suffice it to say that it leads to an underestimation of the placebo effect.) Unlike Beecher, Hróbjartsson and Gøtzsche found that placebos have very small average effects. A problem with their analysis was heterogeneity: they compared apples and oranges. ${ }^{7}$ They included any placebo for any condition in any type of patient. These different placebo interventions have been shown to have different effects. For example, sham injections and sham devices are more effective than placebo pills. ${ }^{89}$ In addition, the colour, ${ }^{10}$ and perceived cost can influence placebo effects. ${ }^{11}$ Hence, Hróbjartsson and Gøtzsche may have been correct that average placebo effects are small, but they are quite powerful for some common ailments, especially pain. ${ }^{7}$

Several studies have now started to investigate the magnitude of the nocebo effects, and are committing the same error as Beecher. We identified 20 systematic reviews have investigated adverse event rates within placebo groups within clinical trials. ${ }^{12}$ On average, half of the placebo-treated patients report an adverse event, and 5\% drop out due to alleged intervention-induced intolerance. ${ }^{12}$ Most of the adverse events identified are pain-related, but they also include depression, restless leg syndrome, and worsening of symptoms from Parkinson's disease, multiple sclerosis, cardiovascular disease, and, motor neuron disease. ${ }^{12}$ Many of these studies refer to adverse event rates within patients who have taken placebos as nocebo effects. ${ }^{13-15}$ Yet, most patients enroll in clinical trials because of some underlying condition that usually have symptoms. Moreover, many common 'symptoms' such as mild to moderate pain, nausea, headaches, arise in most people in their daily lives. Such symptoms may be misattributed to the study intervention, including the placebo (see Figure 2, bottom). To address this, I and a team of researchers used a similar method to the one used 
Howick J. Unethical informed consent caused by overlooking poorly measured nocebo effects Journal of Medical Ethics Published Online First: 16 February 2020. doi: 10.1136/medethics-2019-105903

by Hróbjartsson and Gøtzsche and compared adverse event rates in placebo groups with adverse event rates in nocebo groups. ${ }^{12} \mathrm{We}$ found that adverse event rates within placebo groups were $53 \%$ greater than adverse event rates within untreated groups (6.51\% versus $4.25 \%)$. The nocebo effects included diarrhea, headache, nausea, rash, asthma symptoms, bleeding, and upper respiratory tract infection. ${ }^{12}$ The additional adverse events measured in the placebo-treated patients were genuine nocebo effects as opposed to responses after taking placebos. Future estimates of nocebo effects should use this method and avoid misattribution.

\section{[Figure 2 about here]}

In an example of nocebo effects in clinical practice, one study recently found an association between countries with higher rates of statin intolerance and the number of Google sites describing statin adverse events in those countries. ${ }^{16}$ Readers who have had routine contact with the medical profession may relate to what my colleague Dan Moerman reports:

Before I had my first cataract surgery, maybe 15 years ago, I had to read and sign a long, detailed, death-defying statement which was not simply 'scary,' it was terrifying; among many others, I might go blind. I had nightmares for weeks before the surgery. Just last week, I had to read and sign a statement that extracting an infected tooth from my lower jaw 'might break my jaw.' I confess that afterward, I felt as if I had been in a serious fistfight, and had lost. But, no broken jaw. ${ }^{17}$

Moerman's nightmares and, most likely, at least part of his jaw pain, were nocebo effects caused by the communication of intervention risks within clinical practice.

Underlying mechanisms of the nocebo effect have been studied at a biological level. Expecting a negative outcome leads to increased stress hormones, ${ }^{18-20}$ and lower levels of beneficial endogenous substances such as dopamine. ${ }^{21}$ Nocebo mechanisms have been studied most extensively in the context of pain. Anticipating a negative outcome can augment pain transmission through cholecystokinin receptors, ${ }^{22}$ which can induce pain. ${ }^{19}$ Indeed, experimentally induced negative expectations engage neural circuits that modulate the perception of touch, pressure, pain, and temperature. ${ }^{23}$ The expectation of pain has been associated with similar electromagnetic brain reactions as actual pain, ${ }^{24}$ and expecting pain triggers activation of cholecystokinin, which facilitates pain transmission. ${ }^{23}$ Importantly, pain and (the extent of) tissue damage do not always go hand in hand. Soldiers injured on the battlefield react very differently to the same physical trauma, ${ }^{25}$ and some men experience empathetic labour pain. ${ }^{26}$ These studies demonstrate that psychological causes of pain often overlap with the physical ones, so that the pain we experience arises because of a mix of 
Howick J. Unethical informed consent caused by overlooking poorly measured nocebo effects Journal of Medical Ethics Published Online First: 16 February 2020. doi: 10.1136/medethics-2019-105903

physical and psychological causes, all of them producing experienced pain. ${ }^{27} 28$ In short, the nocebo effect is real, and its underlying mechanisms understood. Taking this into account, we can now be more precise about our definition of the nocebo effect:

Nocebo effect: adverse event arising due to beliefs or expectations of a patient, in routine practice or clinical trials. Nocebo effects are not misattributed symptoms.

\section{DISTINGUISHING ADVERSE EVENTS RELATED TO THE EXPERIMENTAL INTERVENTION FROM NOCEBO EFFECTS}

In addition to distinguishing misattributed symptoms from nocebo effects, it is also important to distinguish nocebo effects from adverse events caused by (non-placebo) interventions. For example, if a patient who is in a coma is given methylphenidate hydrochloride, there is a risk of weight loss.

Weight loss in such a patient, should it arise, is an adverse event that is not a nocebo effect (see Figure 2 , middle). Other adverse events are nocebo effects. Consider the case of negative selfcommunication reported in the BMJ in 1995:

A builder aged 29 came to the accident and emergency department having jumped down on to a $15 \mathrm{~cm}$ nail. As the smallest movement of the nail was painful he was sedated with fentanyl and midazolam. The nail was then pulled out from below. When his boot was removed a miraculous cure appeared to have taken place. Despite entering proximal to the steel toecap the nail had penetrated between the toes: the foot was entirely uninjured. ${ }^{29}$

The pain and anxiety experienced by the builder had nothing to do with tissue damage caused by the intervention (the nail), but entirely to do with his (self) communication that the nail had penetrated his foot.

Some adverse events are partly nocebo effects and partly caused by a non-placebo intervention (or, is a nocebo effect in some patients and an adverse event caused by the active intervention in others). In a trial of aspirin versus sulfinpyrazone in the treatment of unstable angina, half the patients were told about possible gastrointestinal side effects. This resulted in a 6-fold increase in the number of these subjects withdrawing from the trial due to minor, subjective alleged gastrointestinal problems. ${ }^{30} \mathrm{It}$ seems likely, or at least plausible, that both the drugs (aspirin and sulfinpyrazone) and the communication about adverse event led to some of the adverse events reported in this trial. 
Howick J. Unethical informed consent caused by overlooking poorly measured nocebo effects Journal of Medical Ethics Published Online First: 16 February 2020. doi: 10.1136/medethics-2019-105903

I will make one final distinction, between direct and indirect nocebo effects. Some patients who are warned about adverse may not experience them but may have increased anxiety due to the worry about such effects arising. Anxiety is correlated with adverse events such as pain ${ }^{31}$ and recovery from infection, ${ }^{32}$ so it can increase the intensity of the adverse event (see Figure 2, middle). Another type of indirect nocebo effect can arise in carers. Consider again the comatose patient discussed above. The weight loss, should it arise, is not a nocebo effect for the comatose patient. However, if the risk of the adverse event is communicated in an unempathic way with the family members or carers of the comatose patient, it may increase their anxiety and stress. I take the increased anxiety and stress within the family members and carers to be an (indirect) nocebo effect. Taking this into account, we can now expand our definition of the nocebo effect:

Nocebo effect: adverse event arising due to beliefs or expectations of a patient, in routine practice or clinical trials, to be distinguished from (a) misattributed symptoms, and (b) adverse events caused by a non-placebo intervention. Nocebo effects can be direct or indirect.

Now that the confusions surrounding the existence of nocebo effects have been cleared up (which was a prerequisite for discussing the ethics of nocebo effects ${ }^{33}$ ) we can discuss the ethics of nocebo effects.

\section{ETHICS: NOCEBO EFFECTS AND INFORMED CONSENT}

The ethics of informed consent has focused almost exclusively on the need to share intervention risks with patients to respect their autonomy. The notion that how such information is shared can cause harm and thus violate the principle of non-maleficence, has not been adequately explored. Towards the end of their classic 1986 textbook on informed consent (A History and Theory of Informed Consent), Faden, Beauchamp, and King mention the anxiety that might arise because patients do not understand the informed consent process. However, the potential harms caused by sharing intervention risk information (in the wrong way) are not mentioned. Whereas the word 'autonomy' is mentioned 152 times in their book, the word 'non-maleficence' is not mentioned at all. This is not a failing of Faden, Beauchamp, and King. At the time they wrote the book there was no research I am aware of that quantified the magnitude of the nocebo effect. Additionally, they were writing at a time when the Nuremberg trials and paternalism were recent memories, making autonomy a necessary focus. This has started to change. In 2009 Corrigan et al. edited a volume that explored many of the subtleties lying at the intersection between sociological, ethical, and legal factors related to informed consent. ${ }^{34}$ In 2015, Alfano argued that the process of informed consent is an intervention, which could, among other things, induce nocebo effects. ${ }^{35}$ In 2018, Gigorov pointed out that, at least in the 
Howick J. Unethical informed consent caused by overlooking poorly measured nocebo effects Journal of Medical Ethics Published Online First: 16 February 2020. doi: 10.1136/medethics-2019-105903

case of pain, one cannot separate informing a patient about the risk of pain, and 'actual' pain. ${ }^{36}$ Also in 2018, Howick et al. used the correct method to distinguish nocebo effects from misattribution. ${ }^{12}$ Clinical epidemiologists are also starting to take note of the nocebo effect. ${ }^{20}$ We are now in a position to make more certain claims about how the existence of nocebo effects affects the ethics of informed consent.

\section{Even Autonomy is Violated by Ignoring Nocebo Effects}

Even autonomy - the putative reason for sharing intervention risks with patients — may be compromised by ignoring nocebo effects. Autonomy dictates that patients who do not want such information should not have such information forced upon them. Yet, such forcing seems common. It is now a requirement for most publicly funded trials in the United Kingdom (UK) that patient representatives assist with the development, design, conduct, and dissemination of clinical trials. In the trials I have been involved with, this has involved meetings with patient representatives. One way we engage is by sharing the patient information leaflets (PILs), which describe the intervention risks (and other trial information) with these representatives, to get their feedback. Patient representatives inevitably make suggestions to make it 'plainer' English (academics are usually bad at plain English), and often note that how the risks are described is too 'scary'. We then modify the patient information leaflet to reflect what the patient representatives suggest and send it back to the ethics committee (sometimes called 'institutional review boards', or IRBs). Then, the ethics committee usually rejects most of our proposed changes and insists that we revert to more technical language and present the risks in ways the representatives insisted were 'scary'.

The ethics committee's insistence is understandable from a medico-legal perspective. They are concerned about 'outlier' cases where a patient might they were not sufficiently well informed about the potential harm, which they experienced. The problem with this approach is that it violates the other patients' right to choose how to be communicated with - their autonomy. Changing the ethics committee's worries may require that they be equally scared of a court case where a patient decided not to enrol in a trial because of the scary way in which risks were presented and subsequently sued for missing an opportunity. I hope this paper addresses this problem without court cases. Violating autonomy by force-feeding risk information to patients shows that the debate about presenting risk information requires balancing different aspects of autonomy with other ethical principles.

\section{Ignoring Nocebo Effects Violates Autonomy and Justice}


Howick J. Unethical informed consent caused by overlooking poorly measured nocebo effects Journal of Medical Ethics Published Online First: 16 February 2020. doi: 10.1136/medethics-2019-105903

Presenting intervention risks in a way that induces unnecessary nocebo effects may also violate beneficence and justice. Patients who are scared away from participating in a trial or taking an intervention due to how possible risks are presented are missing an opportunity to benefit. Likewise, trials often fail because of difficulties in recruiting or retaining patients. Presenting intervention risks in a balanced way that minimizes nocebo effects seems likely improve recruitment and retention rates. Improving recruitment and retention would improve justice by wasting less money wasted on trials that failed to recruit or retain patients. A failed trial may also have harmed patients who were devoted their time to a failed exercise.

\section{REDUCING UNNECESSARY NOCEBO EFFECTS}

Some nocebo effects are inevitable, others can be avoided by replacing unnecessarily negative communication with positive empathic communication. ${ }^{37}$ I will now suggest several ways in which manipulating communication about intervention risk can improve the ethics of informed consent in clinical practice and clinical trials. The following are suggestions based on preliminary evidence, and some can be implemented immediately. However, because the science of reducing nocebo effects is nascent, more research is required to develop others.

\section{What they don't know won't hurt them}

An obvious way to avoid nocebo effects that are dependent on communication is to simply avoid communicating intervention risk information, and there are some cases in which it may be unethical to share intervention risks. I can report an example from personal experience. As a competitive international rower, I developed a stress fracture of some sort in my rib. It was so painful I could barely breathe, let along train properly. And I had an important race coming up. I went to see my doctor who prescribed Diclofenac. He told me it was quite good for relieving pain and inflammation and prescribed it. He didn't tell me that the listed adverse events include vomiting, diarrhea, headache, constipation, fatal heart attack or stroke, as well as stomach or intestinal bleeding. ${ }^{38} \mathrm{Had}$ my doctor warned me about these side effects, it is likely I would have experienced one or more of the milder ones. And even if I had not experienced the adverse event, I would very likely have been anxious about the possibility that I might experience such an event thus inducing a 'secondary' adverse event. Importantly, for the conclusions I will draw from this case, the doctor had regular contact with me so could have managed any adverse events that arose, I was in great shape and extremely resilient so less likely to experience adverse events. As it happens, I did not experience any adverse events, my pain dropped dramatically, and I went on to win a major race in Boston a week later. I believe it would have unethical for my doctor to have shared the risks (which I never experienced). I imagine — and hope — that healthcare practitioners 'at the coalface' make these kinds 
Howick J. Unethical informed consent caused by overlooking poorly measured nocebo effects Journal of Medical Ethics Published Online First: 16 February 2020. doi: 10.1136/medethics-2019-105903

of sensible decisions about how to share information all the time. I also believe that they are acting ethically.

More commonly than cases where it could be unethical to share risk information, there are likely to be cases when withholding information about trial risks is justified if it benefits patients and avoids harm. ${ }^{39}$ In the example of warning patients about gastrointestinal side-effect, harm was avoided (in some patients) by not sharing adverse event risk information. It is beyond the scope of this paper to discuss whether the avoided harms suffice to outweigh the presumed reduced autonomy. Instead, my claim here is the more modest one that such a weighing needs to be done before asserting that it is ethically required to share information about all intervention risks.

\section{Personalised informed consent}

There are many different methods and theories about informed consent, ${ }^{40}$ and evidence that patients often do not understand the processes. ${ }^{41}$ 'Personalised informed consent' is a method that may increase patient understanding and also reduce nocebo effects in many patients. This requires that informed consent be negotiated between the healthcare practitioners and patients. ${ }^{40} 42$ Personalised consent has been used in clinical trials, ${ }^{43}$ as well as clinical practice. ${ }^{40}$ Oxman, Chalmers, and Sackett describe a hypothetical conversation with a patient involving personalised informed consent:

Most patients seem to get less information from their doctors than they want, but others would rather not be told some of the things that some doctors assume that they must want to know. Because you and I don't know each other yet, I'm going to need your help in learning how much information you want about your problem, and about the possible treatment options. I'm going to depend on you to prompt me to give you more information if you think I'm not being sufficiently forthcoming, or to tell me that you've heard enough if you think I'm overdoing it ... Does that seem to you to be an acceptable way of proceeding? ${ }^{40}$

As a result of such conversations, some patients may wish to have risk intervention information repeated often. For this subset of patients, nocebo effects may be increased. However, this subset of patients (the 'worried well') also seem likely to learn about all intervention risks on their own by researching on the internet, so personalisiong consent may not make much of a difference. More importantly, I am not arguing here that nocebo effects should be avoided altogether, but rather that unnecessary nocebo effects should be avoided. The personalised approach will almost certainly reduce unnecessary nocebo effects among patients who do not insist on being repeatedly told about all intervention risks, no matter how unlikely or mild. And even for the 'worried well', an empathic practitioner could share all intervention risks in ways that reduce anxiety. 


\section{Positive framing}

Information about intervention risks can be either positively or negatively framed, without changing the content of the information. In one recent example, 203 healthy adult volunteers were all given a placebo pill described as 'a well-known tablet available without prescription'. They were randomised to either receive a patient information leaflet (PIL) with standard side effect risk information (e.g., 'Common, 1 in 10 people will be affected') or positively framed wording ('Uncommon, 90\% of people will not be affected'). Those who received the positively framed PIL reported fewer sideeffects ( $n=40$ versus $n=55$ ). Other studies have reported similar findings. ${ }^{44}$ Full disclosure about the risks is conveyed in both types of frames. However, the positive frame reduces the incidence of nocebo effects, so it might be introduced as standard.

\section{Patient-friendly leaflets}

Our recent study (unpublished data) found that the information about drug side effects within PILs was often not presented in a balanced way. Harms of trial participation were usually very prominent, and benefits were sometimes not mentioned at all. Future research investigating ways to develop PILs that contain the information required to satisfy autonomy, yet avoid causing nocebo effects, can and should be developed.

\section{CONCLUSIONS AND IMPLICATIONS FOR CLINICAL PRACTICE}

The nocebo effect has received very little attention compared with the placebo effect. Mistaken claims about its magnitude, and failure to consider the ethical implications of its existence, persist. Because they harm patients, efforts to reduce nocebo effects are an ethical requirement. Future studies that estimate nocebo effects need to use untreated control groups and some studies have started to do this. Current informed consent processes (in clinical trials and clinical practice) risk violating the ethical principles of autonomy, beneficence, non-maleficence, and justice. Personalised consent, positive framing, and patient-friendly information leaflets are promising and deserve more attention.

\section{REFERENCES}

1. Reeves RR, Ladner ME, Hart RH, et al. Nocebo effects with antidepressant clinical drug trial placebos. Gen Hosp Psychiatry 2007;29(3):275-7. doi:

10.1016/j.genhosppsych.2007.01.010 [published Online First: 2007/05/09] 
2. Faden RR, Beauchamp TL, King NMP. A history and theory of informed consent. New York ; Oxford: Oxford University Press 1986.

3. Howick J. Questioning the methodologic superiority of 'placebo' over 'active' controlled trials. Am J Bioeth 2009;9(9):34-48. doi: 10.1080/15265160903090041

4. Howick J. Why it can be Ethical to Use Placebos In Clinical Practice. In: Papanikitas A, Spicer J, eds. A Handbook of Primary Care Ethics. Abingdon: CRC Press 2017.

5. Beecher HK. The powerful placebo. J Am Med Assoc 1955;159(17):1602-6.

6. Krogsbøll LT, Hróbjartsson A, Gøtzsche PC. Spontaneous improvement in randomised clinical trials: meta-analysis of three-armed trials comparing no treatment, placebo and active intervention. BMC medical research methodology 2009;9:1. doi: 10.1186/1471-2288-9-1 [published Online First: 2009/01/07]

7. Howick J, Friedemann C, Tsakok M, et al. Are treatments more effective than placebos? A systematic review and meta-analysis. PLoS One 2013;8(5):e62599. doi: 10.1371/journal.pone.0062599

8. de Craen AJ, Tijssen JG, de Gans J, et al. Placebo effect in the acute treatment of migraine: subcutaneous placebos are better than oral placebos. J Neurol 2000;247(3):183-8.

9. Kaptchuk TJ, Stason WB, Davis RB, et al. Sham device v inert pill: randomised controlled trial of two placebo treatments. BMJ 2006;332(7538):391-7.

10. de Craen AJ, Roos PJ, Leonard de Vries A, et al. Effect of colour of drugs: systematic review of perceived effect of drugs and of their effectiveness. $B M J$ 1996;313(7072):1624-6.

11. Waber RL, Shiv B, Carmon Z, et al. Commercial features of placebo and therapeutic efficacy. JAMA 2008;299(9):1016-7. doi: 10.1001/jama.299.9.1016 [published Online First: 2008/03/06]

12. Howick J, Webster R, Kirby N, et al. Rapid Overview of Systematic Reviews of Nocebo Effects in Clinical Trials. Trials 2018;forthcoming

13. Mitsikostas DD, Chalarakis NG, Mantonakis LI, et al. Nocebo in fibromyalgia: metaanalysis of placebo-controlled clinical trials and implications for practice. Eur $J$ Neurol 2012;19(5):672-80. doi: 10.1111/j.1468-1331.2011.03528.x

14. Mitsikostas DD, Mantonakis L, Chalarakis N. Nocebo in clinical trials for depression: a meta-analysis. Psychiatry Res 2014;215(1):82-6. doi: 10.1016/j.psychres.2013.10.019 [published Online First: 2013/11/12]

15. Mitsikostas DD, Mantonakis LI, Chalarakis NG. Nocebo is the enemy, not placebo. A meta-analysis of reported side effects after placebo treatment in headaches. Cephalalgia 2011;31(5):550-61. doi: 10.1177/0333102410391485

16. Khan S, Holbrook A, Shah BR. Does Googling lead to statin intolerance? Int J Cardiol 2018;262:25-27. doi: 10.1016/j.ijcard.2018.02.085

17. Moerman D. Nocebo effects. In: Howick J, ed., 2019.

18. Benedetti F, Amanzio M, Vighetti S, et al. The biochemical and neuroendocrine bases of the hyperalgesic nocebo effect. J Neurosci 2006;26(46):12014-22. doi: 26/46/12014 [pii]

10.1523/JNEUROSCI.2947-06.2006 [published Online First: 2006/11/17]

19. Bingel U, Wanigasekera V, Wiech K, et al. The effect of treatment expectation on drug efficacy: imaging the analgesic benefit of the opioid remifentanil. Science translational medicine 2011;3(70) doi: 10.1126/scitranslmed.3001244 [published Online First: 2011/02/18]

20. Wartolowska K. The nocebo effect as a source of bias in the assessment of treatment effects. F1000Res 2019;8:5. doi: 10.12688/f1000research.17611.2 [published Online First: 2019/08/01] 
21. Scott DJ, Stohler CS, Egnatuk CM, et al. Placebo and nocebo effects are defined by opposite opioid and dopaminergic responses. Arch Gen Psychiatry 2008;65(2):22031. doi: 10.1001/archgenpsychiatry.2007.34 [published Online First: 2008/02/06]

22. Benedetti F, Amanzio M, Maggi G. Potentiation of placebo analgesia by proglumide. Lancet 1995;346(8984):1231. [published Online First: 1995/11/04]

23. Colloca L. Nocebo effects can make you feel pain. Science 2017;358(6359):44. doi: 10.1126/science.aap8488 [published Online First: 2017/10/07]

24. Lorenz J, Hauck M, Paur RC, et al. Cortical correlates of false expectations during pain intensity judgments--a possible manifestation of placebo/nocebo cognitions. Brain Behav Immun 2005;19(4):283-95. doi: 10.1016/j.bbi.2005.03.010 [published Online First: 2005/05/14]

25. Faxon N, Beecher HK. The relief of suffering. In: Faxon N, ed. The hospital in contemporary life. Cambridge MA: Harvard University Press 1949:70-107.

26. Bohren MA, Berger BO, Munthe-Kaas H, et al. Perceptions and experiences of labour companionship: a qualitative evidence synthesis. Cochrane Database Syst Rev 2019;3:CD012449. doi: 10.1002/14651858.CD012449.pub2 [published Online First: 2019/03/19]

27. Hechler T, Endres D, Thorwart A. Why Harmless Sensations Might Hurt in Individuals with Chronic Pain: About Heightened Prediction and Perception of Pain in the Mind. Frontiers in Psychology 2016;7:1638.

28. Ongaro G, Kaptchuk TJ. Symptom perception, placebo effects, and the Bayesian brain. Pain 2019;160(1):1-4. doi: 10.1097/j.pain.0000000000001367 [published Online First: 2018/08/08]

29. Fisher JP, Hassan DT, O’ CN. Minerva. BMJ 1995;310(70) doi: https://doi.org/10.1136/bmj.310.6971.70

30. Myers MG, Cairns JA, Singer J. The consent form as a possible cause of side effects. Clin Pharmacol Ther 1987;42(3):250-3. doi: 0009-9236(87)90143-3 [pii] [published Online First: 1987/09/01]

31. Colloca L, Benedetti F. Nocebo hyperalgesia: how anxiety is turned into pain. Curr Opin Anaesthesiol 2007;20(5):435-9. doi: 10.1097/ACO.0b013e3282b972fb

00001503-200710000-00007 [pii] [published Online First: 2007/09/18]

32. Ojard C, Donnelly JP, Safford MM, et al. Psychosocial stress as a risk factor for sepsis: a population-based cohort study. Psychosom Med 2015;77(1):93-100. doi: 10.1097/PSY.0000000000000120 [published Online First: 2014/12/04]

33. Uusitalo S, Howick J. Philosophy of too much medicine conference report. J Eval Clin Pract 2018;24(5):1011-12. doi: 10.1111/jep.13000

34. Corrigan O. The limits of consent : a socio-ethical approach to human subject research in medicine. Oxford; New York: Oxford University Press 2009.

35. Alfano M. Placebo Effects and Informed Consent. Am J Bioeth 2015;15(10):3-12. doi: 10.1080/15265161.2015.1074302 [published Online First: 2015/10/21]

36. Gigorov N. Telling the Truth About Pain: Informed Consent and the Role of Expectation in Pain Intensity. American Journal of Bioethics Neuroscience 2018;9(3):173-82. doi: https://doi.org/10.1080/21507740.2018.1496163

37. Howick J, Moscrop A, Mebius A, et al. Effects of empathic and positive communication in healthcare consultations: a systematic review and meta-analysis. $J$ R Soc Med 2018:141076818769477. doi: 10.1177/0141076818769477

38. Durbin K. Diclofenac: Drugs.com; 2019 [cited 20198 October]. Available from: https://www.drugs.com/diclofenac.html accessed 8 October 2019. 
39. Foddy B. A duty to deceive: placebos in clinical practice. The American journal of bioethics : AJOB 2009;9(12):4-12. doi: 10.1080/15265160903318350 [published Online First: 2009/12/17]

40. Oxman AD, Chalmers I, Sackett DL. A practical guide to informed consent to treatment. BMJ 2001;323(7327):1464-6. [published Online First: 2001/12/26]

41. Tam NT, Huy NT, Thoa le TB, et al. Participants' understanding of informed consent in clinical trials over three decades: systematic review and meta-analysis. Bull World Health Organ 2015;93(3):186-98H. doi: 10.2471/BLT.14.141390

42. Doyal L. Good clinical practice and informed consent are inseparable. Heart 2002;87(2):103-6. doi: 10.1136/heart.87.2.103 [published Online First: 2002/01/18]

43. Cresswell P, Gilmour J. The informed consent process in randomised controlled trials: a nurse-led process. Nurs Prax N Z 2014;30(1):17-28. [published Online First: 2014/05/21]

44. Barnes K, Faasse K, Geers AL, et al. Can Positive Framing Reduce Nocebo Side Effects? Current Evidence and Recommendation for Future Research. Front Pharmacol 2019;10:167. doi: 10.3389/fphar.2019.00167 [published Online First: 2019/03/22]

Figure 1. References in Pubmed to the terms 'placebo' and 'nocebo' (any field)

Figure 2. Nocebo effects distinguished from other causes of adverse events 